\title{
Design of Electronic Stethoscope and Heart Rate Monitor for Remote Area Application
}

\author{
V.R. Prasad \\ Electronics \& Telecommunication Engineering \\ SITRC, Nasik \\ Maharashtra, India
}

\author{
G.M. Phade \\ Electronics \& Telecommunication Engineering \\ SITRC, Nasik \\ Maharashtra, India
}

\begin{abstract}
Developing countries like India, where doctor to patient ratio is around 1:1700, as large number of population lives in villages and remotely located area. Health check-up of such a large population is very challenging for their government due to less number of qualified doctors, highly qualified doctor refuges to work in villages and remotely placed areas. This paper proposes a new idea by which an electronic stethoscope is used to provide health check-up of such large population, by creating a public health center (PHC) in every village or combining more small villages to one PHC and connecting it with authorized center, authorized center monitors more than fifteen PHC. Recording of human organ signal by using stethoscope and transmitted to the authorized center using electronic mail can reduce the requirement of large number of doctors.
\end{abstract}

\section{General Terms}

Signal transmission, Authorized center, Recording and Analysis of signal, Internet connectivity.

\section{Keywords}

Stethoscope, PHC, Arduino board, Electronic mail.

\section{INTRODUCTION}

Highly populated countries like India where doctors to patients ratio is very low, due to low doctors to patient ratio, very poor health check-up of needy and poor people residing in remote area and villages. Qualified doctor refuges to work in remote area, unavailability of qualified doctors in remote area and villages, death ratio is very high due to illness and disease in these areas. Work carried out in this paper, uses a electronic stethoscope [1-2] designed with the help of arduino microcontroller board, that records human organ signals, stored in memory [9-10], which is transmitted to the authorized center where qualified doctors analyze signal receive from the particular public health center (PHC). Qualified doctors analyze signal and if any illness present in signal then necessary medicine is prescribe to patients through local doctor.

Figure 1 illustrates that how signal processed by the processor at the primary health center stored in the memory and sent to the nearest authorized center for the further analysis purpose, it also demonstrate that how signal from nearest authorized center is received by the local doctor at PHC, it is simply done by electronic mail[3-4] as it is simple to send and receive the signal very efficiently and safely[11], data consume by signal captured from patient body contain few Mega-Bytes of data, it is efficient to use electronic mail. Every PHC is connected with some kind of transmission media like high-speed Broadband or mobile tower with high-speed internet connectivity, it is possible to send and receive signal.

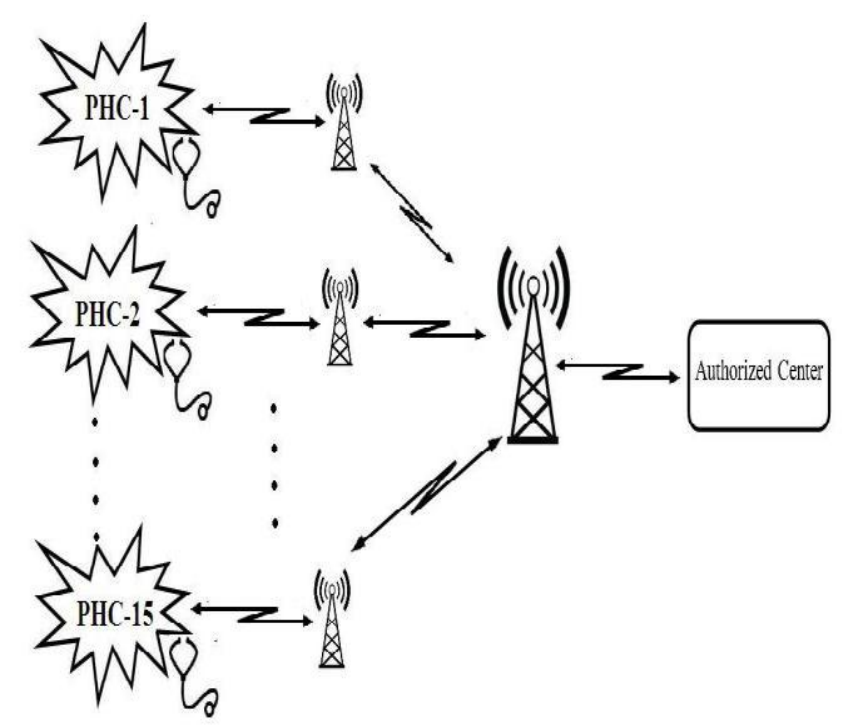

Fig 1: Signal Transmission

\section{HARDWARE DESIGN}

Figure 2 shows circuit diagram of an electronic stethoscope designed with the help of (ATMEGA-328 microcontroller) arduino uno [5] processor. Signal captured from human organ using stethoscope is given to the audio amplifier for signal amplification, LCD and keypad is also interface for the user interaction with stethoscope. The amplifier LM358, which amplifies the signal taken from human organ with analog sensor, applied to the Arduino board for processing of signal. Keypad 20x4 is connected to the arduino board for user command, LCD display is also embedded with arduino board for viewing heart beats, simple programming is used for communicating arduino board with other interface equipments. After storing signal at PHC, it 


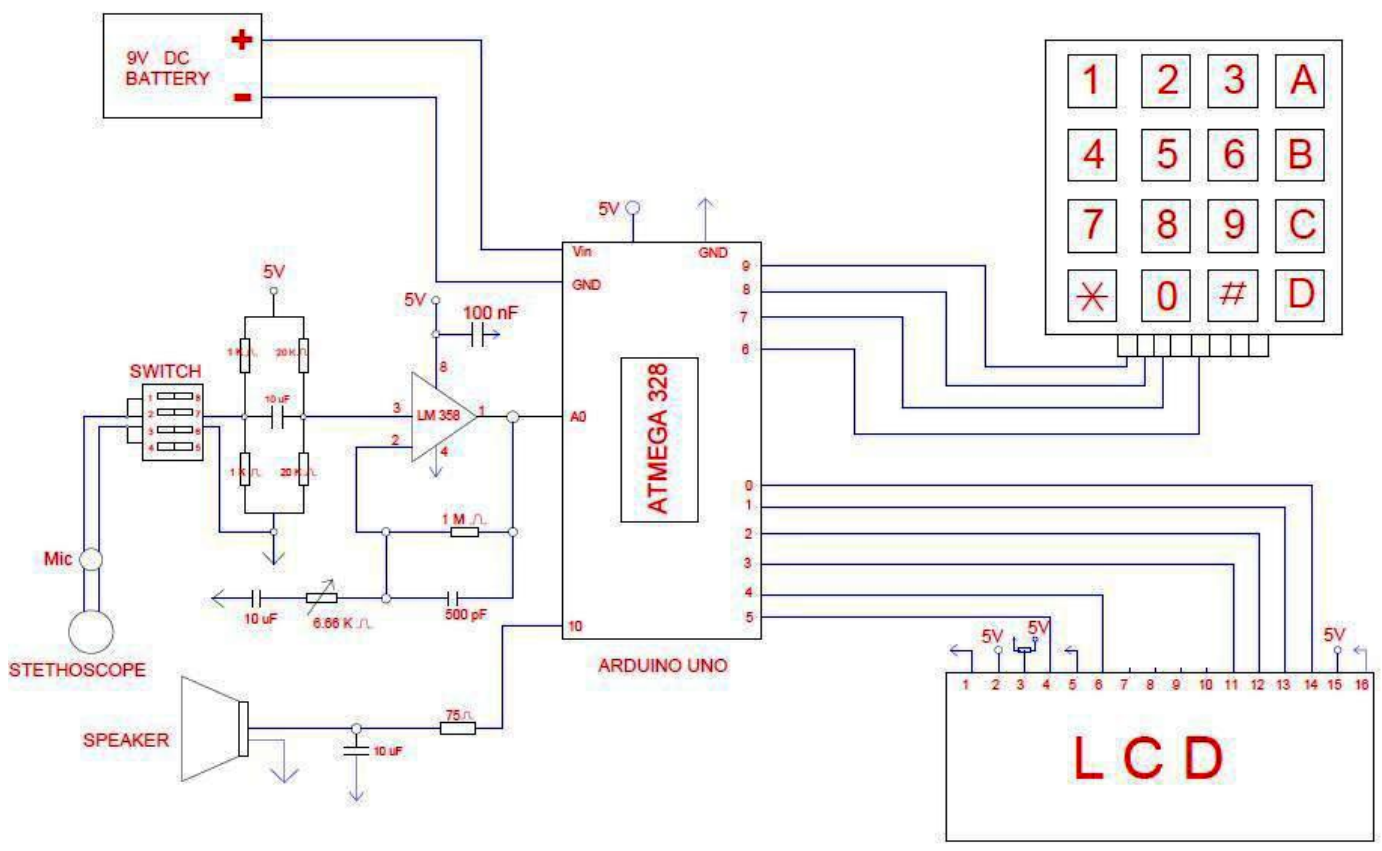

Fig 2: Electronic Stethoscope Circuit Diagram

transmitted via electronic mail to the nearest authorization center and after analysis it is received to local PHC. Every time same procedure is followed and by using this method communication between local PHC, authorization center is established.

\section{RESULT AND DISCUSSION}

\subsection{Analysis of Signal after Recording}

Recording of data (vibration occur when stethoscope is placed on human body) by using stethoscope and stored in memory, used for further analysis[13-14][16]. Recorded signal is send to authorization center by using electronic mail, where expert doctor use various software to plot these signal for analysis. All PHC can communicate in the similar fashion with authorization center for sending of such data recorded with the help of stethoscope.

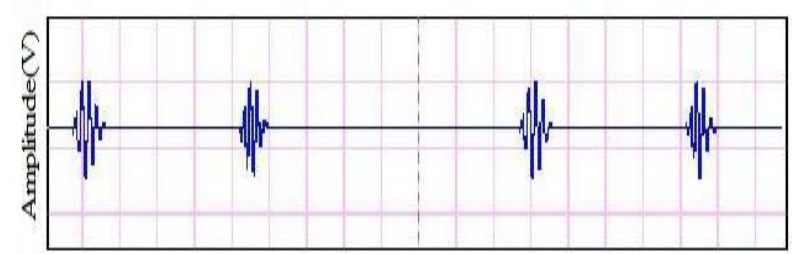

$\operatorname{Tim}(S)$

Fig 3: Heart Sound as a Input

Data recorded is stored in the (.wav) file format as it is suitable to plot or analyze further. As shown in figure 3 heart sound with .wav format is applied as a input and output is recorded on the CRO. Signal is not much stronger(less amplitude of few mili-volt) to detect illness present in that signal, therefore this signal is applied to power amplifier to enhance the signal for further use shown in figure 4.

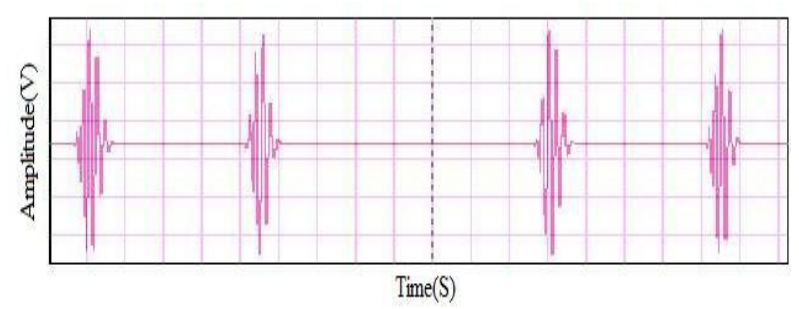

Fig 4: Heart Sound after Amplifier

Different types of lungs sound like normal vesicular lung sound, respiratory stridor lung sound, coarse crack-less lung sound, pleural friction lung sound and wheezing lung sound. Most of the time with the use of traditional stethoscope, weak signal is not detected and that leads to major problem. As shown in figure 5 normal vesicular lung sound, which is not visible clearly as it has very low amplitude when it is detected with electronic stethoscope. Hence cannot be analyzed clearly, therefore it is applied to power amplifier to enhance the signal as shown in figure 6 .

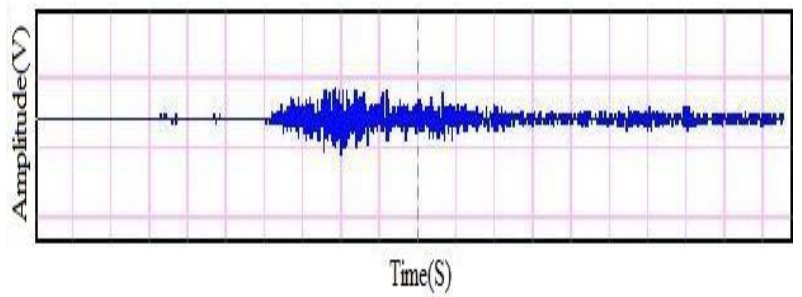

Fig 5: Normal Vesicular Lung Sound as Input 


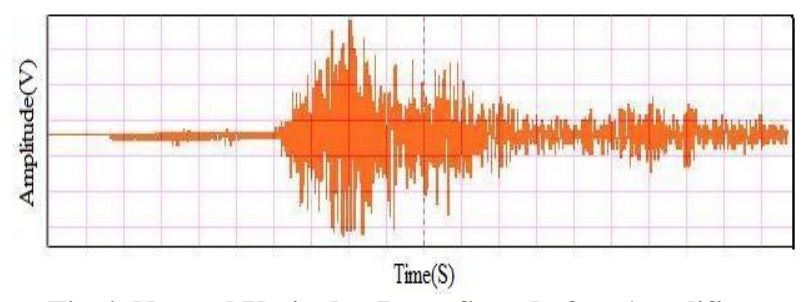

Fig 6: Normal Vesicular Lung Sound after Amplifier

Using this technique a electronic stethoscope is able to record signal and which can be analyzed by doctors, with the help of various software it is easy to analyze illness present in the signal.

\subsection{Heart Beats Monitoring}

Heart beats monitoring, basically used for monitoring heart beats [6-8] in beats per minute (BPM). For this purpose, Arduino processor is used in this paper. As shown in figure 7 when power is switched on first initialization message is printed on LCD screen.

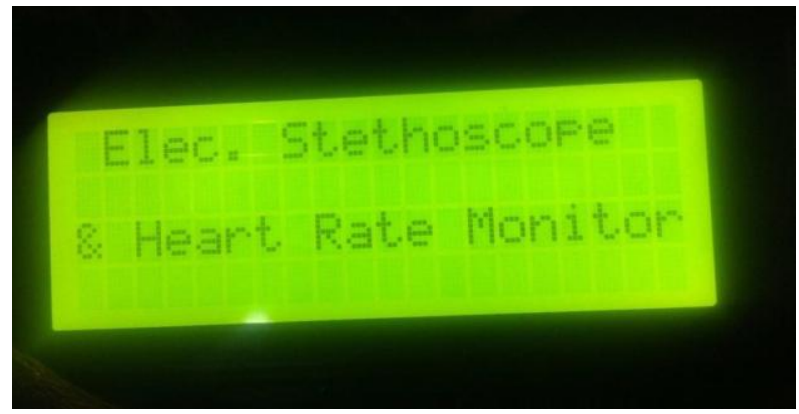

Fig 7: Initialization message

Interfacing of keypad with arduino processor enables user to choose a particular key on the keypad, by choosing a key, user can select mode for which interval of time data, user wants to record. After initialization message on LCD screen, second message is printed showing user command to select particular interval, he wanted to select. As shown in figure 8 second message is printed on the screen showing press key for different interval of time, as per the need of user, key can be pressed. It up to the user requirement, what duration user wants to record data.

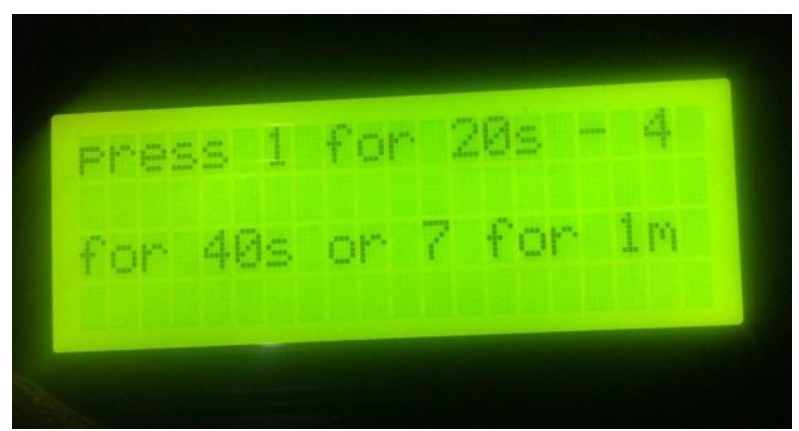

Fig 8: Message for User to select key

If user want to record data for 20 seconds, user can press "key 1 ", as shown in figure 9 screen for 20 second is printed, in this interval of time user record data for normal illness like cough, cold etc.

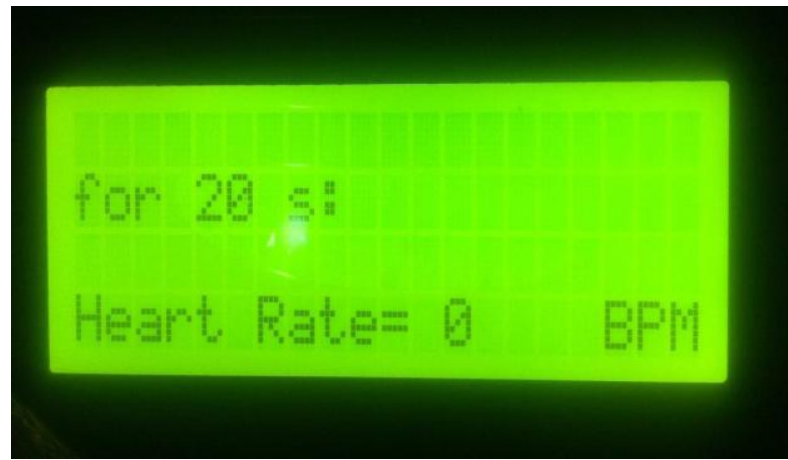

Fig 9: Recording for 20 Seconds

User can press "key 2" for recording data for 40 seconds, as shown in figure 10 when key 2 is pressed after that this screen appears, in this interval of time user can record data for some major illness like systolic murmur, which can lead to sudden death of patient.

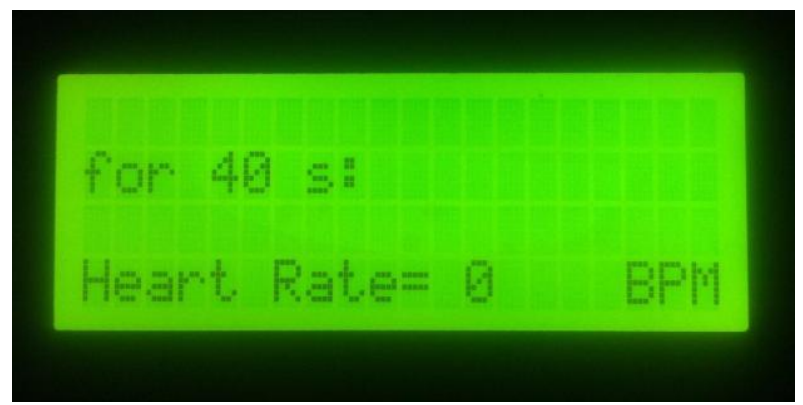

Fig 10: Recording for 40 Seconds

If users want to record data for longer time i.e. around one minute, user can press "key 7" which can record data for one minute. As shown in figure 11 when "key 7" pressed at that time this screen appears, in this interval of time data recorded can be used for detecting major problem which require longer time to detect, like problem in intestine, listening internal heart sound and measuring blood pressure etc.

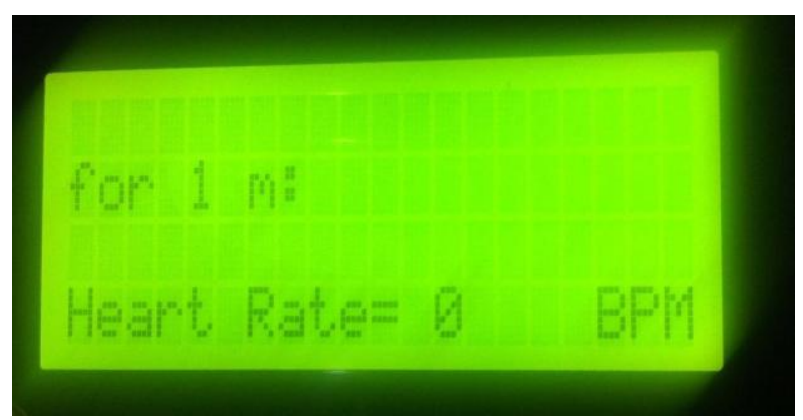

Fig 11: Recording for one Minute

Table 1 shows memory requirement for recording of data for particular time of interval. When key ' 1 ' is pressed signal can be recorded for 20 seconds, consuming memory of 6.8MB(.wav file), when key ' 4 ' is pressed it record data for 40 seconds, that consume memory of $11.4 \mathrm{MB}$ (.wav file), similarly when key ' 7 ' is pressed, it will consume $16.5 \mathrm{MB}$ of memory for recording 1 minute of data. 
Table 1. Result of Heart Beats Calculation

\begin{tabular}{|c|c|c|c|}
\hline Key & Duration & $\begin{array}{c}\text { Memory } \\
\text { (.wav file) }\end{array}$ & Remark \\
\hline $\mathbf{1}$ & $20 \mathrm{Sec}$ & $6.8 \mathrm{MB}$ & $\begin{array}{c}\text { Less Duration, } \\
\text { Useful for normal } \\
\text { signal detection. }\end{array}$ \\
\hline $\mathbf{4}$ & $40 \mathrm{Sec}$ & $11.4 \mathrm{MB}$ & $\begin{array}{c}\text { Long Duration, } \\
\text { Capable of } \\
\text { detecting major } \\
\text { problem. }\end{array}$ \\
$\mathbf{7}$ & $1 \mathrm{Min}$ & $16.5 \mathrm{MB}$ & $\begin{array}{c}\text { Longer Duration, } \\
\text { Detection of } \\
\text { internal heart sound, } \\
\text { B.P. }\end{array}$ \\
\hline
\end{tabular}

As memory consumption is up to $15 \mathrm{MB}$ (.wav file), It is possible to use electronic mail for sending data to PHC authorization center. All these duration indicates different time interval used for detection of various illness. Using arduino processor, a simple low power, portable electronic stethoscope[12][15][17] is designed. Different command given by user, data can be recorded and sent to authorization center for analysis. Suitable for medical check-up for remotely located people.

\section{CONCLUSION}

Designing a cost effective electronic stethoscope with an arduino uno microcontroller, as it does not contain any costlier component, with the use of this electronic stethoscope, medical check-up of remotely located and people who lives in villages will be possible where doctor to patients is very low. This method uses less experience or less qualified doctor, who will attend the PHC and takes reading using electronic stethoscope and transmit it to authorization center for analysis by expert doctors, in this way one authorization center can communicate with more than ten to fifteen PHC. Using this technique government can provide medical checkup to poor and needy peoples, reducing death ratio due to lack of medical assistance.

\section{ACKNOWLEDGMENTS}

The authors would like to thank the reviewers for their careful reading and giving constructive comments. V. R. Prasad would like to thank the entire members of Sandip Institute of Technology \& Research Center (SITRC), family members and those who knowingly \& unknowingly have contributed in their own way in completion of this paper.

\section{REFERENCES}

[1] Bishop, P.J.,"Evolution of the Stethoscope," Journal of the Royal Society of Medicine, pp 448-456.

[2] JingPing Xu, L.G. Durand,"Nonlinear transient chirp signal modeling of the aortic and pulmonary components of the second heart sound," IEEE Trans-actions on Biomedical Engineering .
[3] Yi Luo,"Portable Bluetooth Visual Electrical Stethoscope Research," 11th International Conference on Communication Technology Proceeding(ICCTP), 2008.

[4] Yang Tang, Guitao Cao, Hao Li, "The design of electronic heart sound stethoscope based on Bluetooth," 4th International Conference on Bioinformatics and Biomedical Engineering (ICBBE), 2010, pp.1-4.

[5] Abdullah A. A.,"Simply Arduino," Cairo: CCBY3.0, 2012.

[6] Habin Wang, Jian Chen, Choi Samjin,"Heart Sound Measurement And Analysis Sys- tem with Digital Stethoscope," International Conference on Biomedical Engineering and Informatics, 2009.

[7] A Harshola, S.Thale, M.S.Panse, "Digital Stethoscope for Heart Sounds,"Proceedings published by International Journal of Computer Applications(IJCA), 2011, pp 4245 .

[8] Brusco M., and Nazeran H.,"Development of an intelligent pda-based wearable digital phonocardiograph,'In Proceedings of the 2005 IEEE Engineering in Medicine and Bi-ology 27th Annual Conference.

[9] Samuel Wilks, "Evolution of the stethoscope,"Popular Science, vol.22, no.28, pp.488-491, Feb 1883 ISSN 0161-7370.

[10] Liu Ping, Peng Aiming, Peng Puping,"A Novel Electrocardio Signal Detection and Analysis System Based on Virtual Instruments,"8th International Conference on Electronic Measurement and Instruments, 2007.

[11] Ying-Wen Bai and Chao-Lin Lu,"Web-based Remote Digital Stethoscope,"9th Interna-tional Conference on Internet and Multimedia System, 2005.

[12] Shin J.Y., Lim S.W., Kim Y.C., Kim S.J., Cha E.J., Lee T.S., "Portable digital esophageal stethoscope system ", Annual International Conference of the IEEE, Publication Year: 2010, pp 1844-1847.

[13] Jatupaiboon N., Pan-ngum S., Israsena P.,"Electronic stethoscope prototype with adaptive noise cancellation", Knowledge Engineering, 2010, 8th International Conference on ICT, Publication Year: 2010 , pp 32-36.

[14] Udawatta L., Abeykoon A.H.S., Prasanga D.K., Prasad S., Perera W., Perera K.,"Knowledge on heart patients through stethoscopic cardiac murmur identification for E-healthcare," Knowledge Engineering, 2010 8th International Conference on ICT. Publication Year: 2010, pp 58-63.

[15] Jia-Ren Chang Chien, Cheng-Chi Tai, "The implementation of a Bluetooth-based wireless phonocardio-diagnosis system,"Networking, Sensing and Control, 2004 IEEE International Conference. Volume: 1 Publication Year: 2004, pp170-173 Vol.1.

[16] Samuel E. Schmidt, Egon Toft, Claus Holst-Hansen, Johannes J. Struijk, "Noise and the detection of coronary artery disease with an electronic stethoscope," 20105 th Cairo International Biomedical Engineering Conference Cairo, Egypt, December 16-18, 2010 pp 54- 56.

[17] Christian McMechan and Poman So,"Design and Implementation of a Low Cost Electronic Stethoscope," IEEE714-718. 